\title{
Hadroproduction of quark flavors
}

\author{
Francis Halzen \\ Department of Physics, University of Wisconsin, Madison, Wisconsin 53706 \\ Satoshi Matsuda ${ }^{\dagger}$ \\ Rutherford Laboratory, Theory Division, Chilton, Didcot, Oxon, England
}

(Received 19 September 1977)

\begin{abstract}
We show that the extension of the conserved-vector-current hypothesis to gluons successfully describes the magnitude and energy dependence of the hadroproduction of strangeness; charm, and $b, t$ flavors in their manifest $(K, D, B)$ or hidden $(\phi, \psi, \Upsilon)$ forms. We investigate the relation between the hadronic excitation of $\psi$ and $\Upsilon$ particles for different assumptions regarding the quark structure of $\Upsilon$. In our approach cross sections are simply obtained by rescaling experimental data on the production of virtual photons by $\alpha_{g}^{2}(M) / \alpha^{2}$, where $\alpha_{g}(M)$ is the quark-gluon coupling constant, varying with the gluon mass $M$ in the sense of asymptotic freedom. We propose the powerful phenomenological tool that the total inclusive yield for producing flavor bound states scales in $\Gamma / M^{3}$ where $M$ and $\Gamma$ are respectively the mass and direct hadronic width of the state. We also show that the model successfully describes the relative production rates of dileptons (via $\psi$ as well as "off-resonance") for different beam particles.
\end{abstract}

\section{INTRODUCTION AND SUMMARY}

It is the purpose of this paper to present a simple picture, unifying in a quantitative way the experimental features of the hadroproduction of heavyquark flavors. Our basic assumption ${ }^{1,2}$ is that pairs of heavy quarks [having strangeness, charm, or the quark flavor(s) associated with $\Upsilon(9.5)$ (Ref. 3 )] originate from a hadronic collision via a single gluon and that the cross section for producing a gluon of mass $M$ is determined by the measured cross sections for producing a virtual photon with the same $M$ via the conserved-vector-current hypothesis ${ }^{4}$ (CVC) (see Fig. 1). Our proposal is to extend the CVC hypothesis, which relates the hadroproduction of the electromagnetic (virtual photons) and weak ( $W, Z$ bosons) currents, to gluons. The model introduces a complete symmetry between leptons and quarks regarding their production mechanism in hadron collisions (see Fig. 2), a symmetry that has been a powerful tool in unraveling the spectroscopy of quarks and leptons.

The model has the big advantage of being free of parameters; it nevertheless correctly correlates a wide variety of data. We summarize below how it accommodates the prominent features of hadroproduction of heavy-quark flavors. The predictive power and simplicity of this idea have to be contrasted to previous attempts to "explain" the same features by introducing two or more mechanisms, each with considerably more freedom of phenomenological adjustments ${ }^{5,6}$ : (i) We will show that, given the experimental data on the production of lepton pairs, the CVC hypothesis correctly accounts for the magnitude and energy dependence of the production of stangeness, charm, and $b, t$ flavors $\mathrm{s}^{7}$ in their manifest $(K, D, B)$ or hidden $(\phi$, $\psi, \Upsilon$ ) forms. (ii) It allows us to understand the observed similarity in longitudinal- and transverse-momentum dependence of dileptons produced via resonance $\left(\phi, \psi, \psi^{\prime} \cdots\right)$ or via contínum. As they are given by the same diagram (see Figs. 1 and 2) $\left\langle p_{T}\right\rangle$ and $\langle x\rangle\left(x=2 p_{L} / \sqrt{s}\right)$ of dileptons should indeed smoothly interpolate between continuum photons and resonances $\left(\phi, \psi, \psi^{\prime} \cdots\right)$. (iii) As single (colored) gluons materalize into $\phi, \psi, \Upsilon$. states via the emission of two soft gluons or a soft gluon and a photon, the se states are singly

a)

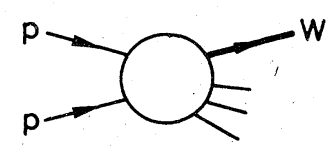

b)

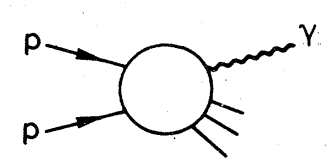

c)

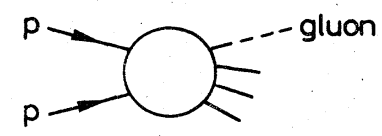

$a_{g}\left(Q^{2}\right)$

FIG. 1. Schematic representation of the extension of the conserved-vector-current hypothesis to the gluon current, producing quark flavors in hadron collisions via the materialization of gluons into quark-antiquark pairs. 
produced. The Okubo-Zweig-Iizuka (OZI) rule ${ }^{5}$ is not expected to be operative in production processes as $\phi, \psi, \Upsilon, \cdots$ originate via a "Zweig-violating" single gluon. (iv) Finally we will add the assumption that photons and gluons couple to the initial hadron interaction via light $(u, d)$ quark pairs (see Fig. 3) and demonstrate the compatibility of the CVC model with recent data on dilepton production with various beam-particle types.

We will also demonstrate that the general structure of the model yields the result that the production cross sections of flavor-bound states as $\phi, \psi, \psi^{\prime}, \Upsilon$ scale in $\Gamma / M^{3}$, where $M$ and $\Gamma$ are, respectively, the mass and direct hadronic width of the state. The power of this scaling rule as a phenomenological tool can be best illustrated by calculating $^{8,9}$ the production level ${ }^{3}$ of $\Upsilon(9.5)$ by rescaling the experimental $\psi$ cross section. It then follows that

$$
\sigma_{\Upsilon}(\sqrt{s})=\frac{\Gamma_{\Upsilon}}{\Gamma_{\psi}}\left(\frac{M_{\psi}}{M_{\Upsilon}}\right)^{3} \sigma_{\psi}\left(\frac{M_{\psi}}{M_{\Upsilon}} \sqrt{s}\right) .
$$

For a $5-\mathrm{GeV}$ quark mass, potential models ${ }^{10}$ binding quark-antiquark pairs, typically yield ${ }^{11} \Gamma \simeq 10$ $\mathrm{keV}$ and $B_{\Upsilon}=\left[\Gamma\left(\Upsilon \rightarrow \mu^{+} \mu^{-}\right)\right] /[\Gamma(\Upsilon \rightarrow$ all $)] \simeq 0.05$.

Equation (1) can also be rewritten as

$$
B_{\Upsilon} \sigma_{\Upsilon}(\sqrt{s})=\left[\frac{B_{\Upsilon}}{B_{\psi}} \frac{\Gamma_{\Upsilon}}{\Gamma_{\psi}}\left(\frac{M_{\psi}}{M_{\Upsilon}}\right)^{3}\right] B_{\psi} \sigma_{\psi}\left(\frac{M_{\psi}}{M_{\Upsilon}} \sqrt{s}\right)
$$

and relates $B_{\mathrm{r}} \sigma_{\mathrm{r}}(28 \mathrm{GeV})$ via a factor of 3.6 $\times 10^{-3}$ to $B_{\psi} \sigma_{\psi}(9 \mathrm{GeV}) \simeq 10^{-7} \mathrm{mb}$. We therefore correctly obtain $B_{\Upsilon} \sigma_{\Upsilon}(28 \mathrm{GeV}) \simeq 3.6 \times 10^{-10} \mathrm{mb}$. Note, however, that this argument relies completely on the assumption that the activity in the $10-\mathrm{GeV}$ dilepton-mass region is due to a set of narrow (keV) resonances smeared by experimental resolution. It would obviously be very difficult to understand the production level in the $\Upsilon$ region on the basis of resonances with width of order $\mathrm{GeV}$. A detailed discussion of the hadronic production cross sec-
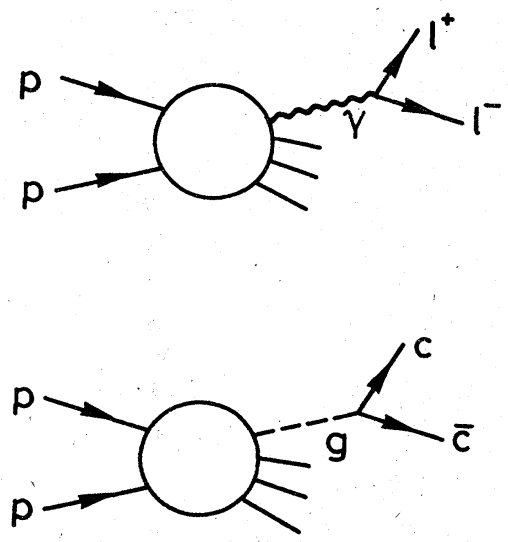

FIG. 2. Symmetry between the production mechanisms of quarks and leptons in hadron collisions:

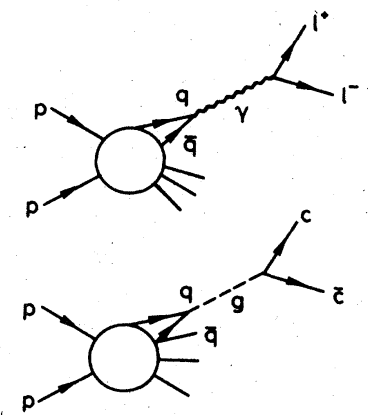

(a)

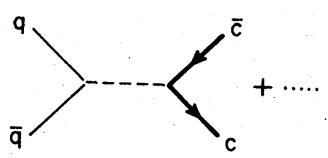

(b)

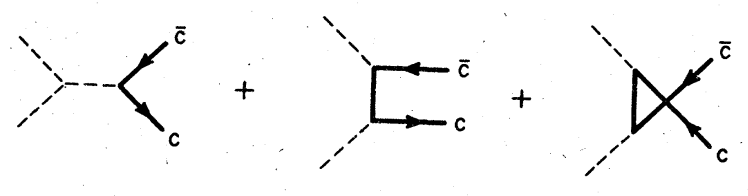

(c)

FIG. 3. (a) Supplementary assumption (cf. Fig. 2) that photons and gluons originate from a hadronic collision via quark-antiquark pairs. (b) Typical single-gluon diagram for producing charmed quarks in lowest order in $\alpha_{g}$. (c) Two-gluon diagrams for producing charmed quarks in lowest order in $\alpha_{g}$.

tion of $\Upsilon$ for various assumptions regarding its quark content will be presented further on (see Fig. 10).

In Sec. II we set up the framework of the model and discuss the mass and energy dependence of hadroproduction of heavy-mass states, focusing in detail on the expected production level of charmed particles. In Sec. III we apply the model to the production of flavor-bound states and reserve Sec. IV for a detailed discussion of dilepton production with different beam-particle types. We conclude in Sec. V with a few comments on the topical problem of hadroproduction of weak bosons.

\section{HADROPRODUCTION OF QUARK FLAVORS}

The production cross section of virtual photons of mass $M$, i.e., $\sigma(p p \rightarrow \gamma(M) X)$, determines 
through the CVC hypothesi $\mathrm{s}^{4}$ the production of the weak neutral boson $Z$ by simply rescaling the electromagnetic cross section $\sigma\left(p p \rightarrow \gamma\left(M=M_{Z}\right) X\right)$ in coupling by a factor $(G / \alpha)^{2}$. We propose ${ }^{1,2}$ that a completely parallel argument (Fig. 1) allows us to relate the production of gluons to that of virtual photons by the relation

$$
\sigma(p p \rightarrow g(M) X)=C \frac{\alpha_{g}{ }^{2}(M)}{\alpha^{2}\left\langle e^{2}\right\rangle} \sigma(p p \rightarrow \gamma(M) X) .
$$

Here $\left\langle e^{2}\right\rangle \simeq 0.2$ is the average charge squared of the quarks participating in the massive photon production and $\alpha_{g}(M)$ is the "running" quark-gluon coupling constant given by asymptotic freedom ${ }^{12}$

$$
\alpha_{g}(M)=\frac{\alpha_{g}\left(M_{0}\right)}{1+(25 / 12 \pi) \alpha_{g}\left(M_{0}\right) \ln \left(M^{2} / M_{0}{ }^{2}\right)} .
$$

The factor $C\left(=\frac{2}{3}\right)$ in Eq. (3) is the color counting relating the production cross sections of lepton and hadron pairs in $q \bar{q}$ annihilations, i.e.,

$$
\left(\sum_{i, j, k, l=1}^{3} \frac{\lambda_{A}^{i j}}{2} \delta^{A B} \frac{\lambda_{B}^{k l}}{2}\right) / \sum_{i, j=1}^{3} \delta_{i j}{ }^{2} .
$$

Notice that this approach extends CVC from flavor to color dimens ions, relating the color-singlet (photon and weak) to color-octet (gluon) current. That the CVC hypothesis could be extended to gluons has been motivated by Fritzsch ${ }^{1}$ in the context of quantum chromodynamics (QCD). On a more naive level one might just argue that the extension is plausible provided $\alpha_{g}(M)<1$ in Eq. (4), allowing for some perturbation in the number of gluons. ${ }^{2}$ This of course would seem to preclude any application to light quarks with perhaps $\alpha_{g}(M)>1$, although it has been suggested that the CV C approach might survive as a lower bound on the production cross sections of light quarks. ${ }^{13,14}$ The extended CVC hypothesis should certainly be justified within the same phenomenological approach $^{15,16}$ that successfully relates the $\phi$ and $\psi$ OZI-violating widths via the well-known threegluon diagram

$$
\Gamma(\psi \rightarrow \text { hadrons })=\frac{3}{2} \frac{M_{\psi}}{M_{\phi}}\left(\frac{\alpha_{g}(3)}{\alpha_{g}(1)}\right)^{6} \Gamma(\phi \rightarrow 3 \pi)
$$

with $\alpha_{g}(1) \simeq 0.55$ and $\alpha_{g}(3) \simeq 0.28$ in agreement with Eq. (4). Note, however, that the diagram where each proton emits a gluon, with a subsequent twogluon annihilation into a gluon has no analog in the electromagnetic case and therefore violates CVC. In lowest order in $\alpha_{g}$ this diagram, along with the two other diagrams of the "two-gluon" type shown in Fig. 3(c), would invalidate our single-gluon approximation if they substantially contributed to the heavy-quark production cross section. The best argument for their actual suppression is phenomenological: experimentally ${ }^{17} \sigma(p p \rightarrow \psi X) /$ $\sigma(p \bar{p}-\psi X) \simeq 0.15$ at $p_{1 \mathrm{ab}}=40 \mathrm{GeV} / c$, precluding any important role of the two-gluon diagrams in the production of charmed quarks as they contribute equally in the $p p$ and $p \overline{\text { cases. }}$

In lowest order in $\alpha_{g}$ (Ref. 18) one can actually evaluate the diagrams of the single-gluon type [e.g., the quark-antiquark annihilation diagram of Fig. 3(b)] and compare them with the two-gluon diagrams. A straightforward calculation yield $\mathbf{s}^{19}$ a suppression factor ${ }^{20}$ of 0.33 in the matrix element for charm production by the set of diagrams of Fig. 3(c) relative to just the first [see Fig. 3(b)] of the set of single-gluon diagrams. The twogluon diagrams are further suppressed by the magnitude of the initial-state gluon wave functions relative to the product of quark-antiquark probability distributions. This will be the case up to Fermilab energies ${ }^{21}$ but any quantitative estimates are difficult and crucially depend on the choice of structure functions.

In the context of QCD, the generalized CVC hypothesis allows us to calculate a lower bound on the production cross section of heavy-quark flavors at all energies. This bound should approximately represent the actual cross section up to an energy, which is difficult to calculate because of our ignorance of the detailed structure of the gluon $x$ distributions, but is situated in the Fermilab range $(\sim 150 \mathrm{GeV} / c)$ for the choice of structure functions of Ref. 21 .

As an example we start by calculating the hadroproduction of $K$ mesons. These are produced as $K^{+} K^{-}$pair $\mathrm{s}^{22}$ originating from a gluon via its materialization into an $s \bar{s}$ quark pair. The mass of the gluons is limited from below by $M=2 M_{K}$ and from above by the total available collision energy $M=\sqrt{s}$. At any $M$ value within this range their cross section is determined by Eq. (3); therefore

$$
\sigma_{K}(\sqrt{s})=C \frac{1}{\alpha^{2}\left\langle e^{2}\right\rangle} \int_{2 M_{K}}^{\sqrt{s}} \alpha_{g}^{2}\left(M^{\prime}\right) \frac{d \sigma^{\gamma}}{d M^{\prime}} d M^{\prime}
$$

or, as the lower limit dominates the integral in Eq. (6)

$$
\sigma_{K}(\sqrt{s}) \simeq C \frac{\alpha_{g}^{2}\left(2 M_{K}\right)}{\alpha^{2}\left\langle e^{2}\right\rangle} \int_{2 M_{K}}^{\sqrt{s}} \frac{d \sigma^{\gamma}}{d M^{\prime}} d M^{\prime} .
$$

Equation (7) can of course be generalized to any new threshold of mass $M$

$$
\sigma_{M}(\sqrt{s})=C \frac{\alpha_{g}^{2}(M)}{\alpha^{2}\left\langle e^{2}\right\rangle} \int_{M}^{\sqrt{s}} \frac{d \sigma^{\gamma}}{d M^{\prime}} d M^{\prime}
$$

exhibiting the fact that, given the experimental virtual photon cross section $d \sigma / d M^{\prime}$ as a function of the photon mass $M^{\prime}$, Eq. (8) predicts the mass dependence of the production cross section of quark flavors. Note also that the energy dependence 


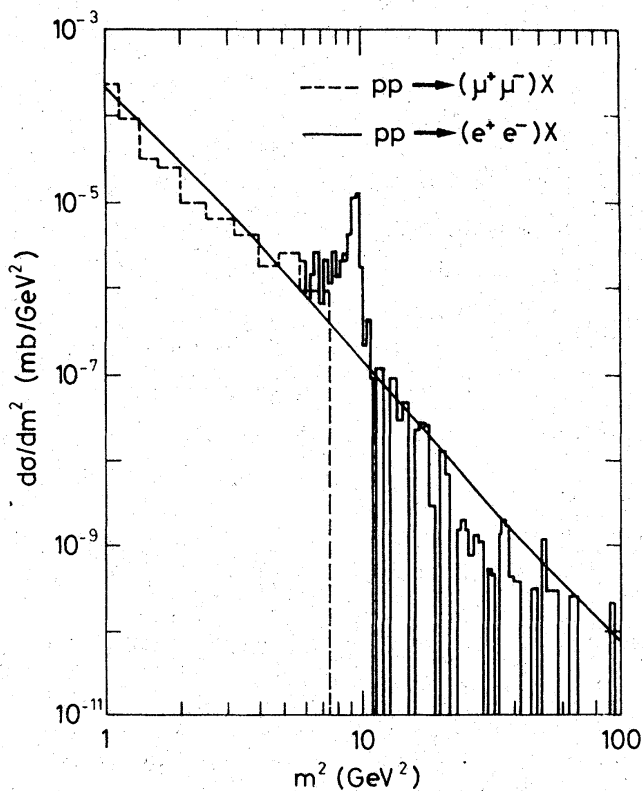

FIG. 4. The production cross section for lepton pairs in $p p$ collisions with $p_{1 \mathrm{ab}}=300-400 \mathrm{GeV} / c$, is plotted as a function of the dilepton mass $M$ along with the parametrization of Eq. F (1). The data (see Ref. 24) have been corrected for the limited acceptance of the experiments and integrated over $y$ and $p_{T}$. We refer for details to S. Chavin and J. D. Sullivan, Phys. Rev. D 13, 2990 (1976); F. Halzen and W. Long, University of $\bar{W}$ isconsin Report No. COO-480, 1976 (unpublished).

of the cross section for producing a given flavor [say strangeness in Eq. (7)] is determined by the scaling of the photon production cross section

$$
\frac{d \sigma^{\gamma}}{d M^{\prime}}=\frac{1}{M^{\prime 3}} F\left(\frac{\sqrt{s}-\sqrt{s_{\text {th }}}}{M^{\prime}}\right) \text {. }
$$

Note that our scaling variable differs from the usual $\sqrt{s} / M^{\prime}$ variable by a correction $\sqrt{s_{\mathrm{th}}}=2 M_{N}$ $+M^{\prime}$ in $p p$ collisions, which becomes important close to production threshold. The use of this scaling variable in the context of the production of heavy particles has been extensively motivated in the past. ${ }^{23}$

By integrating the experimental ${ }^{3,24}$ photon cross sections ${ }^{25,26}$ at $p_{1 a b}=300-400 \mathrm{GeV} / c$, shown in Fig. 4, Eq. (8) yields the general mass dependence of production cross sections in $p p$ collisions. The result is shown in Fig. 5. The horizontal scale has been chosen such that $M=2 M_{K}$ and $2 M_{D}$ correspond to the $K$ and $D$ points, respectively. Note that we correctly calculate the production level of strange particles and that the relative production level of strange $\left(M=2 M_{K} \simeq M_{\Phi} \simeq 1\right)$ and charmed particles $\left(M \simeq 2 M_{D} \simeq M_{\psi} \simeq 3\right)$ is given by

$$
\frac{\sigma_{K}}{\sigma_{D}} \simeq \frac{\alpha_{g}{ }^{2}(1)}{\alpha_{g}^{2}(3)} \frac{\int_{2 M_{K}}^{\sqrt{s}}\left(d \sigma^{\gamma} / d M^{\prime}\right) d M^{\prime}}{\int_{2 M_{D}}^{\sqrt{s}}\left(d \sigma^{\gamma} / d M^{\prime}\right) d M^{\prime}}
$$

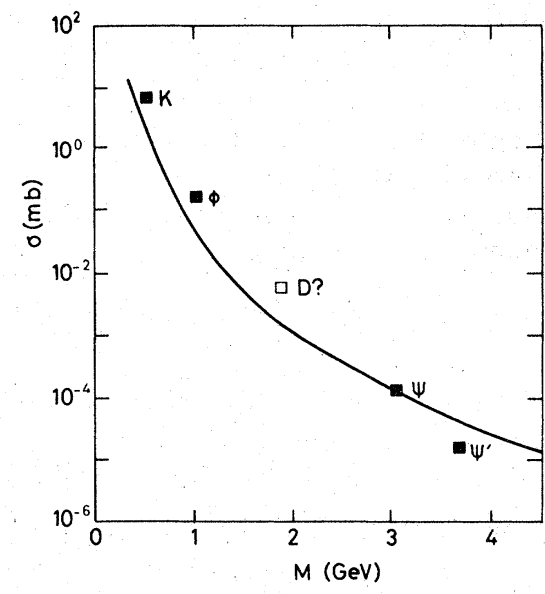

FIG. 5. The total inclusive cross section for producing a particle of mass $M$ in $p p$ interactions is plotted as a function of $M$ at $p_{1 a b}=300 \mathrm{GeV} / c$, along with data (Ref. $24)$ on $K, \phi, \psi$, and $\psi^{\prime}$ production in the vicinity of that energy. Also shown is the inclusive $D$ production cross section as estimated from Eq. (11).

The production cross section for charmed particles around $300 \mathrm{GeV} / c$ is predicted at the $1-\mu \mathrm{b}$ level. This is consistent with expectations from other models, ${ }^{5} e_{\circ} g_{\circ}$, with the argument that

$$
\frac{\sigma(p p \rightarrow D X)}{\sigma(p p \rightarrow \psi X)} \simeq \frac{\sigma(p p \rightarrow K X)}{\sigma(p p \rightarrow \phi X)} .
$$

Equations (8) and (9) with $M=2 M_{D}$ allow us to calculate the energy dependence of $\sigma_{D}$ and therefore to obtain the charm threshold in $p p$ collisions from

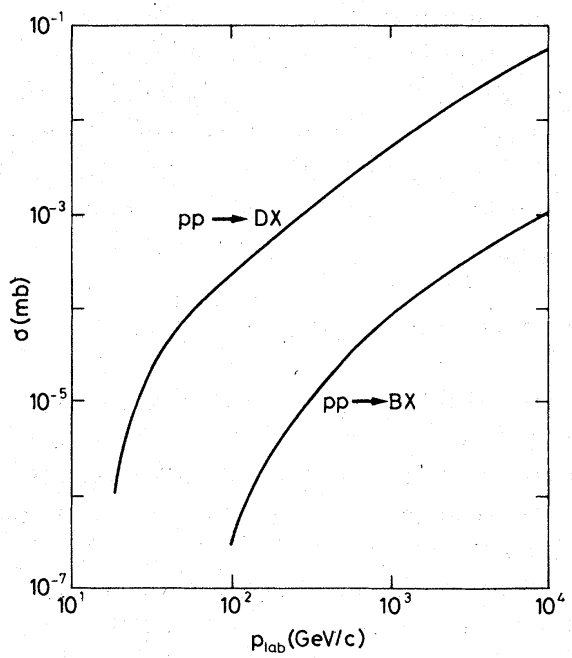

FIG. 6. Energy dependence of the total inclusive cross section for $D(c \bar{u})$ and $B(b \bar{d})$ production. The curves can be approximately parametrized as $\sigma_{M}(\sqrt{s})$ $\simeq\left(5 \times 10^{-3}\right)\left[\alpha_{g}{ }^{2}(2 M) /(2 M)^{4}\right]\left(\sqrt{ } s-2 M_{N}-2 M\right)^{2}$ with $M_{D} \simeq 1.87$ and $M_{B} \simeq 5$. (Units are $\mathrm{mb}$ and GeV.) 


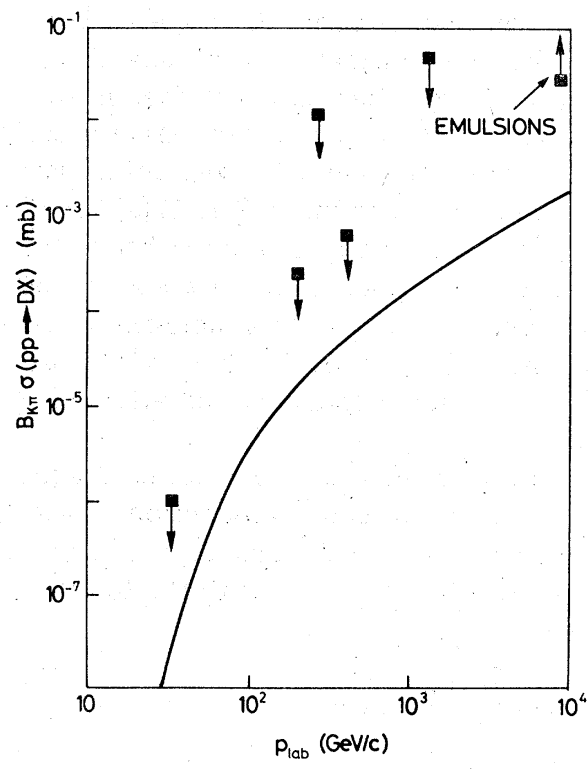

FIG. 7. The total inclusive cross section for charmed-particle production, shown in Fig. 6 , has been multiplied by the $D \rightarrow K \pi$ branching ratio and is compared with experimental upper limits (Ref. 24) as well as to a rough estimate (Ref. 27) of the cross section at $p_{1 \mathrm{ab}} \simeq 10^{4} \mathrm{GeV} / c$ on the basis of evidence from emulsion exposures to cosmic rays.

virtual-photon data. Using the approximate $M^{-5}$ dependence of $d \sigma^{\gamma} / d M^{\prime}$ at $\sqrt{s}=25 \mathrm{GeV}$, we obtain from Eqs. (8) and (9)

$$
\sigma_{D}(\sqrt{s}) \simeq\left(5 \times 10^{-3}\right) \frac{\alpha_{g}^{2}(3)}{\left(2 M_{D}\right)^{4}}\left(\sqrt{s}-2 M_{N}-2 M_{D}\right)^{2}
$$

(units are $\mathrm{mb}$ and $\mathrm{GeV}$ ). The result is shown in Fig. 6. In Fig. 7 we have plotted $B_{K \pi} \sigma_{D}$ along with present experimental upper $\operatorname{limits}^{5}$. (as well as the cross section hinted ${ }^{27}$ at by cosmic-ray emulsions at $\left.E \simeq 10^{4} \mathrm{GeV}\right)$. We have taken $B_{K \pi}=\Gamma(D \rightarrow K \pi) /$ $\Gamma(D \rightarrow$ all $) \simeq 0.02$ as suggested ${ }^{5}$ by the SLAC data.

We finally turn our attention to the production of $B(b \bar{d})$ states, carrying the quantum number bound into $\Upsilon(9.5)$. Their production cross section can be calculated from Eq. (12) after substitution $M_{D} \rightarrow M_{B} \simeq 5 \mathrm{GeV}$ and $\alpha_{g}(3) \rightarrow \alpha_{g}(5) \simeq 0.2$. The result is shown in Fig. 6. Although the production of $B$ particles is reduced by roughly a factor $\frac{2}{3}\left(M_{D}\right)$ $\left.M_{B}\right)^{4}$, its higher mass significantly reduces backgrounds in double-arm searches, and makes its detection not a priori impossible despite its smaller cross section compared with charmed particles. The existence of some favorable branching ratios might make it more accessible than the $D$ meson in hadron collisions.

\section{HADROPRODUCTION OF FLAVOR-BOUND STATES}

As $\phi, \psi, \psi^{\prime}, \Upsilon$, etc., originate via single gluons their production mechanism is the same in momentum structure as that of virtual photons (see Figs. 1-3). This is of course a basic feature of the CVC hypothesis. The model predicts a similar momentum dependence (in $x$ and $p_{T}$ ) for $\phi$, $\psi, \psi^{\prime}, \Upsilon$ production as for virtual photons adjacent in mass. Experimentally it means that no changes in the $x$ or $p_{T}$ dependence of dileptons are expected when the dilepton mass passes through resonance. This has been a well advertised fact ${ }^{24}$ for $\left\langle p_{T}\right\rangle$ and is illustrated for the $x$ dependence in Fig. 8. One notices indeed that the cutoff in $x$, parametrized as

$$
E \frac{d \sigma}{d x} \propto(1-x)^{N},
$$

is the same for $\psi$ production and for the production of virtual photons adjacent in mass to the $\psi$, as expected from this model.

We now proceed to look in detail at the production cross section of flavor-bound states, concentrating on the $\psi$ for which the most precise measurement of its excitation curve in hadron collisions exists. $\psi$ 's originate from gluons materializing into $c \bar{c}$ pairs below charm production threshold $M=2 M_{D}$; therefore

$$
\begin{aligned}
\sigma_{\psi} & \equiv \sigma(p p-\psi X) \\
& =f C \frac{\alpha_{g}^{2}\left(M_{\psi}\right)}{\alpha^{2}\left\langle e^{2}\right\rangle} \int_{2 M_{C} \simeq M_{\psi}}^{2 M_{D}} \frac{d \sigma^{\gamma}}{d M^{\prime}} d M^{\prime}
\end{aligned}
$$

Here $f$ represents the fraction of $c \bar{c}$ pairs materializing into $\psi$ 's. Indeed some of the pairs will form $\psi^{\prime}, P_{c}$, and $\chi$ states; straightforward counting of states $^{1}$ would suggest $f \simeq \frac{1}{10}$. As we previously

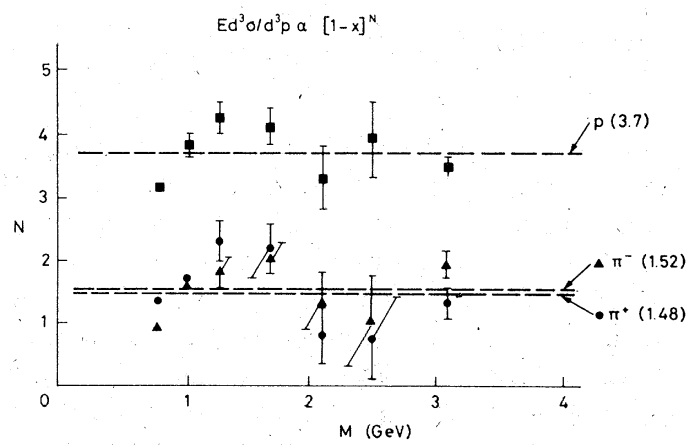

FIG. 8. The longitudinal-momentum dependence of dilepton production in $\pi^{ \pm}$and $p$ collisions on a $C$ target (Ref. 31) has been fitted as $(1-x)^{N}$. The plot shows the dependence of $N$ on the dilepton mass $M$. The average value of $N$ (listed in brackets) is compared with the $N$ value at $M=M_{\psi}$ for the different beam particles. 


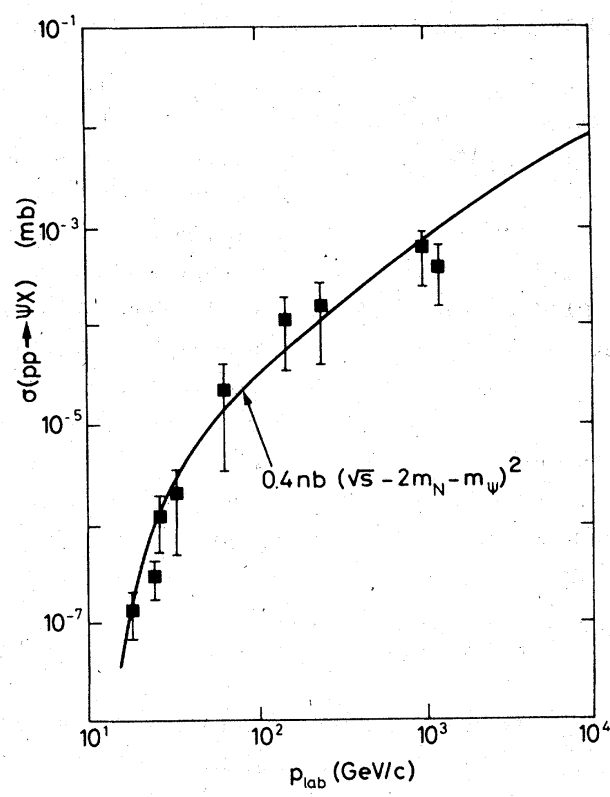

FIG. 9. A comparison with data is made of the calculated dependence of the total inclusive $\psi$ production cross section in nucleon-nucleon interactions on the incident momentum.

described for the case of charmed-particle production, the scaling requirement of $d \sigma^{\gamma} / d M^{\prime}$ fixes the energy dependence of $\sigma_{\psi}$. Substitution of Eq. (F1) of Ref. 25 into Eq. (14) shows that the second term in Eq. (F1) dominates, yielding the result

$$
\sigma(p p \rightarrow \psi X) \simeq\left(0.4 \times 10^{-6}\right)\left[\sqrt{s}-2 M_{N}-M_{\psi}\right]^{2},
$$

in agreement with the data (see Fig. 9). A completely parallel argument allows us to calculate the production cross section of the $\Upsilon(9.5)$ set of resonances

$$
\left.\frac{d \sigma}{d M d y}\right|_{y=0}=\left.f C \frac{\alpha_{g}^{2}\left(M_{\gamma}\right)}{\alpha^{2}\left\langle e^{2}\right\rangle} \int_{8.8}^{10.6} \frac{d \sigma^{\gamma}}{d M^{\prime} d y}\right|_{y=0} d M^{\prime} .
$$

Here we have fixed the integration range in Eq. (16) to correspond to the region of "activity" in the experimental dilepton spectrum. ${ }^{3,28} f$ stands for the fraction of $b \bar{b}$ pairs materializing into $C$-odd states, we take the $\psi$ as a guide to $\operatorname{fix} f \simeq \frac{1}{10}$. The prediction of Eq. (16) is shown in Fig. 10, using the parametrization of Eq. (F2) of Ref. 25 for $(d \sigma /$ $\left.d M^{\prime} d y\right)\left.\right|_{y=0}$ and assuming a typical ${ }^{11}$ leptonic branching ratio $B=0.03 \sim 0.05$. In view of the accumulating assumptions, estimating the observed cross section within a few standard deviations has to be considered successful. We present below an indirect, but much simpler way of calculating the same cross section.
We attempt next an estimate of the factor $f$ describing the materialization of gluons into $\psi$ 's. In QCD a color-octet gluon materializes into a colorsinglet $\psi$ via a two-step process: first a soft gluon is emitted to form a $C$-even quark pair; secondly another soft gluon [Fig. 11(a)] or a photon [Fig. 11(b)] is emitted in order to change the $C$ conjugation of the quark pair. As $\psi$ 's will be produced via even- $C$ states, this mechanism is analogous to the cascade model ${ }^{5}$ where $\psi$ 's originate via production and subsequent radiative decay of $\chi$ states.

Combination of the gluon production diagram of Fig. 1 with the final-state interaction of Fig. 11 yields expressions for the production cross sections of flavor-bound states with the following structure:

$$
\sigma_{M}(\sqrt{s})=\frac{\alpha_{g}{ }^{4}(M)}{M^{2}} H\left(\frac{\sqrt{s}}{M}\right)
$$

or

$$
\sigma_{M}(\sqrt{s})=\frac{\alpha \alpha_{g}{ }^{3}(M)}{M^{2}} H^{\prime}\left(\frac{\sqrt{s}}{M}\right)
$$

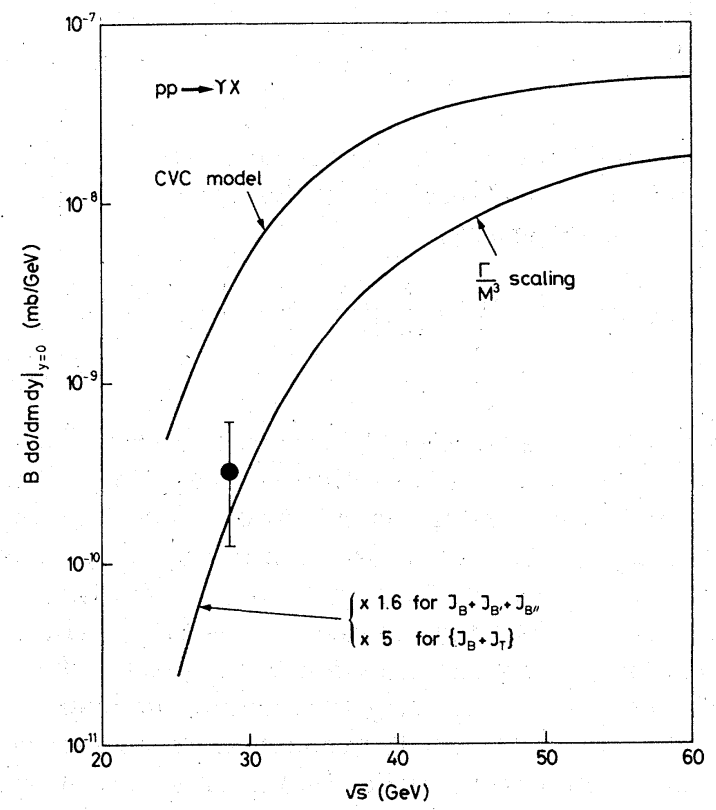

FIG. 10. Inclusive cross section multiplied by the leptonic branching ratio for producing $\Upsilon(9.5)$ in nucleon interactions with $y=0$. The curves correspond to two alternative ways of estimating the result shown: (i) using the CVC model; (ii) by rescaling the $\psi$ data according to the relation of Eqs. (1) and (2). The curve shown has to be enhanced by a factor of 1.6 if one sums over the three expected (Ref. 11) narrow $b \bar{b}$ bound states and by a factor of 5 if six states corresponding to almost degenerate $b \bar{b}$ and $t \bar{t}$ states would be present in the $\Upsilon(9.5)$ dilepton mass region. 


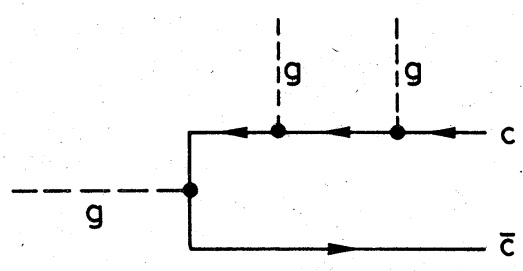

a)

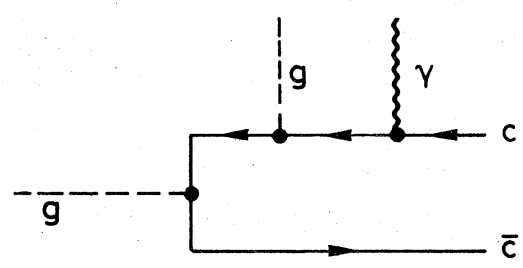

b)

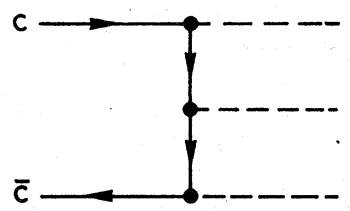

c)

FIG. 11. Typical mechanisms for the materialization of (colored) gluons into $C$-odd $c \bar{c}$ states (e.g., $\psi$ 's): (a) via the emission of two soft gluons; (b) via the emission of a soft gluon forming a $C$-even $c \bar{c}$ state (e.g., $\chi^{\prime} s$ ) and its subsequent radiative transition to a $C$-odd state. The three-gluon diagram describing the direct hadronic width of $c \bar{c}$ bound states below charm threshold is shown in (c).

in cases (a) and (b), respectively. As the direct hadronic width of flavor-bound states calculated from the three-gluon diagram ${ }^{15,16}$ of Fig. 11(c) results in

$$
\Gamma \propto \alpha_{g}{ }^{3} M\left|\psi_{0}\right|^{2}
$$

we obtain from purely dimensional analysis and Eqs. (17), (18), and (19) that

$$
\sigma_{M}(\sqrt{s})=\frac{\alpha_{g}(M) \Gamma}{M^{3}} F\left(\frac{\sqrt{s}}{M}\right)
$$

or

$$
\sigma_{M}(\sqrt{s})=\frac{\Gamma}{M^{3}} F^{\prime}\left(\frac{\sqrt{s}}{M}\right)
$$

provided we can neglect residual $M$ dependence [besides the dimensional dependence made explicit in Eq. (19)] of the (dimensionless) quark-antiquark wave functions in the diagrams of Fig. 11. In practice the scaling rules of Eqs. (17') and (18') are indistinguishable with the present uncertainties on the data. These relations are not absolute anyway as higher-order gluon corrections may enter. We show in Fig. 12 the experimental verification of Eq. $\left(18^{\prime}\right)$ for $\phi, \psi, \psi^{\prime}, \Upsilon$ by plotting the quantity $\left(M^{3} / \Gamma\right) \sigma_{M}(\sqrt{s})$ for the different states. ${ }^{29}$ The scaling of this quantity is nicely exhibited in Fig. 12 .

This test is highly nontrivial as the data points on the graph of Fig. 12 would scatter over more than seven orders of magnitude without rescaling. At this point we remind the reader that Eq. (18') can be recast in the form of Eq. (1) and subsequently used to rescale the $\psi$ cross section in energy to obtain the $\Upsilon$ yield. Using the same $\Gamma$ and $B$ values we obtain the result of Fig. 10 in agreement with the data. The calculated cross section has to be increased by a factor 1.6 if one sums over the three expected ${ }^{11} b \bar{b}$ bound states and by a factor of 5 if $b$ and $t$ quarks are close in mass and six resonances make up the $\Upsilon$ region. Trying to disentangle the charge of the quark hidden in $\Upsilon$ seems premature. The relative production rate of the $b \bar{b}$ states is of course determined by their direct hadronic width. Using the results of Ref. 11 we would obtain relative production ratios for $J_{B}, J_{B}^{\prime}$,

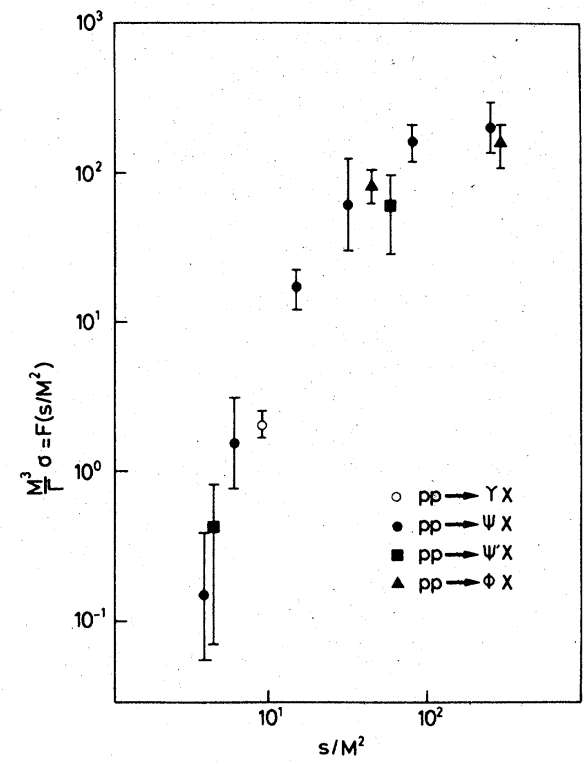

FIG. 12. The figure demonstrates that the quantity $M^{3} / \Gamma \sigma$ is a universal function of $s / M^{2}$ for different flavor-bound states. $M, \Gamma$, and $\sigma$ are, respectively, the mass, direct hadronic width, and total inclusive production cross section in hadron collision of the flavor-bound state. 
$J_{B}^{\prime \prime}$ of about $3: 1: 1$ for the quantity $B \sigma$. We conclude this section by drawing attention to the fact that both calculations of the $\Upsilon$ excitation curve predict a cross section increased by almost two orders of magnitude in the $\sqrt{s} \simeq 50 \mathrm{GeV}$ energy range and above (see Fig. 10).

\section{DILEPTON PRODUCTION WITH DIFFERENT BEAM PARTICLES}

It is the purpose of this paragraph to show that the model in which $\psi$ particles originate in hadron collisions via single gluons finds added support $\mathrm{t}^{30}$ from recent measurements at Fermilab ${ }^{31}$ and the CERN SPS ${ }^{17}$ of $\psi$ production with beams of different particle type. In our model relative production rates of $\psi$ 's are directly related to the relative production rates of virtual photons in the corresponding mass range. In the absence of dilepton data for other beam particle types over a sufficient mass range (similar to the proton data shown in Fig. 4) we compensate for the lack of data by adding the assumption that virtual photons and gluons are produced via the annihilation of quarks and antiquarks (see Fig. 3 ). ${ }^{32}$ The analysis is greatly simplified by limiting $x$ of the produced system to the beam fragmentation region, say $x>0.15$. As $x=x_{1}-x_{2}$, where $x_{1}$ and $x_{2}$ are the longitudinal-momentum fractions of the partons originating, respectively, from the beam and the target, we also limit $x_{1}>0.15$ (see Fig. 13). Therefore only the valence quarks in the beam participate in the reaction, although valence as well as sea quarks in the target have to be taken into account. We will assume a completely symmetric composition of sea quark $\mathrm{s}^{33}$ and parametrize the suppression of sea quarks relative to valence quarks by a parameter $\epsilon$.

As an illustration we calculate the inclusive production of massive photons and $\psi$ 's by a $\pi^{+}(u \bar{d})$ beam on a neutron target (see Fig. 13). We intro-

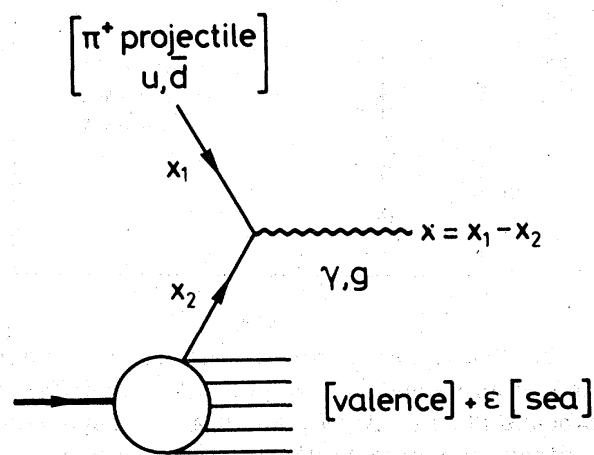

FIG. 13. Schematic presentation of the calculation of beam particle ratios for hadroproduction of dileptons.
TABLE I. $\psi$ production on isoscalar targets. Production ratios of $\psi$ particles on isoscalar targets in the projectile fragmentation region are given as a function of $\epsilon$, the parameter measuring the relative importance of valence and sea quarks in the target. Predictions for $\epsilon=\frac{1}{4}$ (value obtained from the measured $\left.(p / \bar{p})\right|_{\psi}$ ratio) are compared with the data. In the data column, the numbers after the correction $x>0.4$ are given in parentheses, for $p / \bar{p}$ and $K^{+} / K^{-}$.

\begin{tabular}{cccc}
\hline \hline Beams & $\begin{array}{c}\text { Predicted ratios } \\
\text { of } d \sigma / d x(x \geqslant 0.15)\end{array}$ & $\epsilon=\frac{1}{4}$ & Data \\
\hline$\frac{p}{p}$ & $\frac{2 \epsilon}{3+2 \epsilon}$ & 0.14 & $0.15 \pm 0.08^{\mathrm{a}} \rightarrow(0.23)$ \\
$\frac{\pi^{+}}{\pi^{-}}$ & 1 & 1 & $1+0.1^{\mathrm{b}}$ \\
$\frac{K^{+}}{K^{-}}$ & $\frac{4 \epsilon}{3+4 \epsilon}$ & 0.25 & $0.85 \pm 0.5^{\mathrm{a}} \rightarrow(0.24)$ \\
$\frac{p}{\pi^{+}}=\frac{p}{\pi^{-}}$ & $\frac{6 \epsilon}{3+4 \epsilon}$ & 0.38 & $0.4 \pm 0.1\left(\pi^{+}\right)^{\mathrm{b}}$ \\
\hline \hline
\end{tabular}

${ }^{\mathrm{a}}$ Ref. 17. ${ }^{\mathrm{b}}$ Ref. 31.

duce the notation

$$
\begin{aligned}
& \int_{0.15}^{1} d x \frac{d \sigma}{d x}\left(\pi^{+} n-\gamma X\right) \equiv \sigma\left(\pi_{n}^{+}-\gamma\right), \\
& \int_{0.15}^{1} d x \frac{d \sigma}{d x}\left(\pi^{+} n \rightarrow \psi X\right) \equiv \sigma\left(\pi^{+}-g\right) .
\end{aligned}
$$

We obtain

$$
\sigma\left(\pi_{n}^{+} \gamma\right) \propto\left[2 \times\left(\frac{1}{3}\right)^{2}+\epsilon \times\left(\frac{1}{3}\right)^{2}+\epsilon \times\left(\frac{2}{3}\right)^{3}\right] .
$$

The first term corresponds to the annihilation of valence $d \bar{d}$ pairs; the last two terms represent the fusion of $\pi^{+}$valence quarks with the sea quarks in the neutron. For $\psi$ production through a gluon the calculation is identical except for the fact that gluon couplings are independent of the quark changes; therefore,

$$
\sigma\left(\pi_{\vec{n}}^{+} g\right) \propto(2+\epsilon+\epsilon) .
$$

Present experiments use isoscalar ${ }^{31}$ (or approximately isoscalar ${ }^{17}$ ) targets; we therefore have to repeat the calculation for $n$ and $p$ targets and average the cross sections. The results are summarized in Tables I and II. Numerical calculations of the relative production yields of dileptons and $\psi$ 's for different beam particles are also shown in Figs. 14 and 15 as a function of $\epsilon$.

We start the discussion of the data by looking at the results for $\psi$ production (Table I and Figs. 14 and 15). Charge independence yields, independent of the value of $\epsilon$

$$
\left.\frac{\sigma\left(\pi^{+}-\overrightarrow{(I=0)} g\right)}{\sigma\left(\pi^{-}(I=0) g\right)} \equiv \frac{\pi^{+}}{\pi^{-}}\right|_{\psi}=1
$$


TABLE II. Dilepton production on isoscalar targets. Production ratios of massive photons on isoscalar targets in the projectile fragmentation region are given as a function of $\epsilon$, the parameter measuring the relative importance of valence and sea quarks in the target. Predictions for $\epsilon=\frac{1}{4}$ (see Table I) are compared with the data of Ref. 31 .

\begin{tabular}{cccc}
\hline \hline Beams & $\begin{array}{c}\text { Predicted ratios } \\
\text { of } d \sigma / d x(x \geqslant 0.15)\end{array}$ & $\epsilon=\frac{1}{4}$ & Data \\
\hline$\frac{p}{\bar{p}}$ & $\frac{2 \epsilon}{3+2 \epsilon}$ & 0.14 & \\
$\frac{\pi^{+}}{\pi^{-}}$ & $\frac{3+10 \epsilon}{12+10 \epsilon}$ & 0.38 & $0.5 \pm 0.2$ \\
$\frac{K^{+}}{K^{-}}$ & $\frac{5 \epsilon}{6+5 \epsilon}$ & 0.17 & \\
$\frac{p}{\pi^{+}}$ & $\frac{18 \epsilon}{3+10 \epsilon}$ & 0.82 & $0.65 \pm 0.15$ \\
$\frac{p}{\pi^{-}}$ & $\frac{18 \epsilon}{12+10 \epsilon}$ & 0.31 & $0.4 \pm 0.2$ \\
\hline \hline
\end{tabular}

in agreement with both experiments. ${ }^{17.31}$ In order to test the model, one has to measure the $\pi^{+} / \pi^{-}$ ratio on a proton target, where it predicts a ratio $(1+2 \epsilon) /(2+2 \epsilon)$. As $p \rightarrow \psi$ contains no pure valence annihilation contribution, the $p / \bar{p}$ ratio is directly proportional to $\epsilon$. From the observed ${ }^{17}$ value ( $p$ /

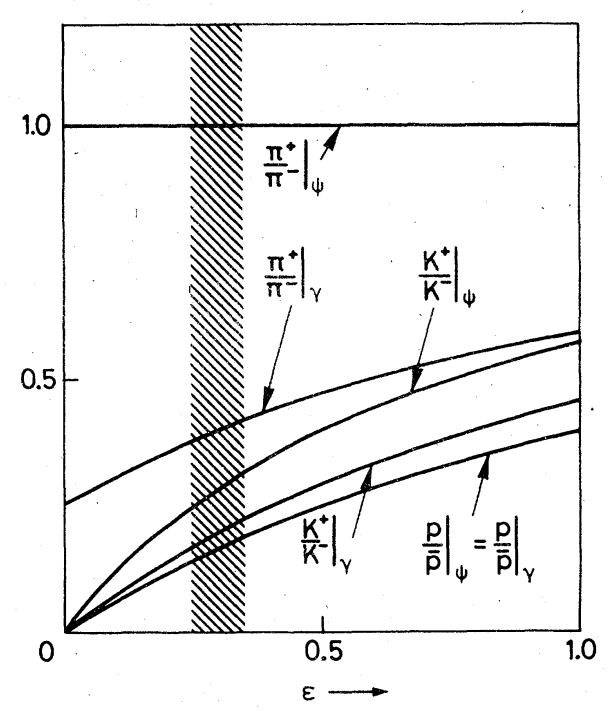

FIG. 14. Production ratios of massive photons and $\psi$ particles on isoscalar targets in the projectile fragmentation region $(x \geq 0.15)$ as a function of $\epsilon$, a parameter measuring the relative importance of valence and sea quarks in the target. The shaded area represents the range of $\epsilon$ values obtained from the measurement (Ref. 17) of $\left.(p / \bar{p})\right|_{\psi}$.

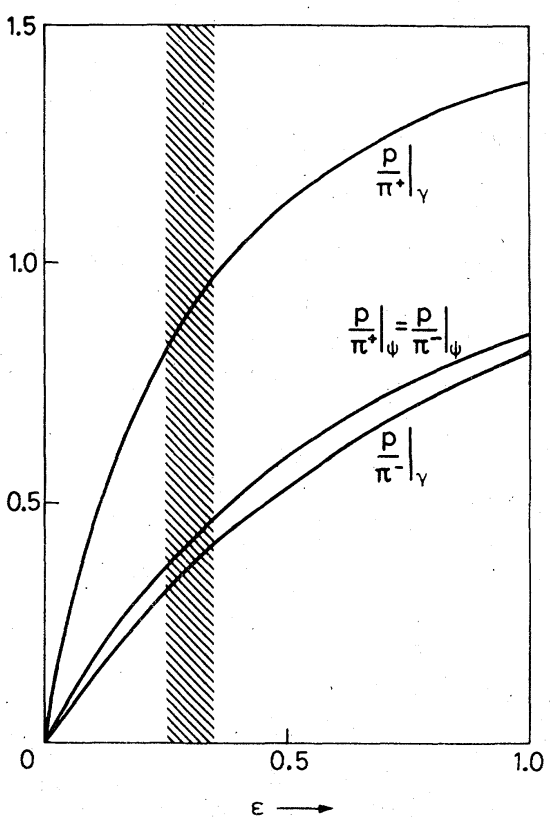

FIG. 15. Same as Fig. 14 for ratios of meson and baryon beams.

р) $\left.\right|_{\psi} \simeq 0.15$, we deduce that $\epsilon \simeq \frac{1}{4}$. The effect of a cut $x>0.4$ on the data would increase this value to about 0.35 . This range of $\epsilon$ values is shown as a shaded area in Figs. 14 and 15. As the errors on the data are large we will for the sake of discussion just use a nominal value $\epsilon=\frac{1}{4}$. We therefore predict

$$
\left.\frac{K^{+}}{K^{-}}\right|_{\psi} \simeq 0.25
$$

This is consistent with the observed ${ }^{17}$ value $(0.85$ $\pm 0.5)$, which is decreased to $0.2-0.3$ by a cut in $x$.

We turn our attention next to photon production (Table II and Figs. 14 and 15). Using the value of $\epsilon$ obtained from the $\psi$ data, we calculate the relative production yields of massive photons. These can be compared with data on dilepton production with mass in the vicinity of the $\psi$ mass, but not on resonance. ${ }^{34}$ We obtain

$$
\begin{aligned}
& \left.\frac{p}{\bar{p}}\right|_{\gamma}=\left.\frac{p}{\bar{p}}\right|_{\psi} \simeq 0.15, \\
& \left.\frac{\pi^{+}}{\pi^{-}}\right|_{\gamma} \simeq 0.38 \quad\left(\mathrm{cf} .\left.\left(\pi^{+} / \pi^{-}\right)\right|_{\psi}=1\right),
\end{aligned}
$$

and

$$
\left.\frac{K^{+}}{K^{-}}\right|_{\gamma} \simeq 0.17
$$

Equation (27) contains the intriguing prediction for the $\pi^{+} / \pi^{-}$ratio that the dilepton yield jumps from 


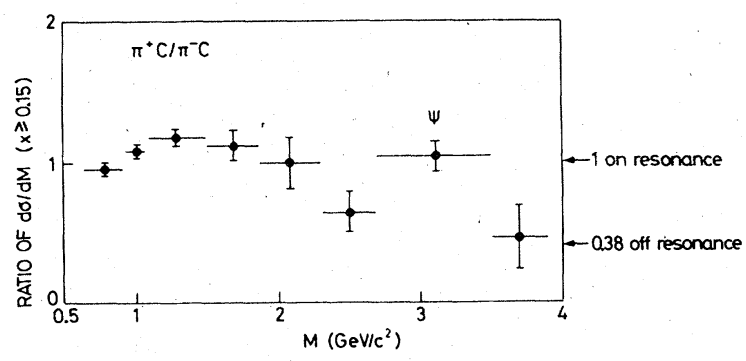

FIG. 16. Comparison of the relation $\left.\left(\pi^{+} / \pi^{-}\right)\right|_{\psi}=1$, $\left.\left(\pi^{*} / \pi^{*}\right)\right|_{\gamma}=0.38$ with the data of Ref. 31. (Data for $M<2.25$ cannot be compared with our predictions because of the presence of the production and subsequent leptonic decay of vector mesons $\rho, \phi, \ldots$.)

0.38 to 1 and back, passing through the $\psi$ resonance. Figure 16 shows that this is indeed the case. The power of these on-off resonance comparisons is also clearly exhibited by comparing $\left.\left(K^{+} / K^{-}\right)\right|_{\gamma}$ and $\left.\left(K^{+} / K^{-}\right)\right|_{\psi}$. Neglecting strange quarks in the sea one predicts equality of the two ratios, whereas the assumption that $s=\bar{s}=\bar{u}$ enhances the $K^{+} / K^{-}$ ratio on resonance by almost a factor of 2 (see Tables I and II). Therefore a $K^{+} / K^{-}$measurement of dileptons as a function of their mass through the $\psi$ region directly measures the strange con-

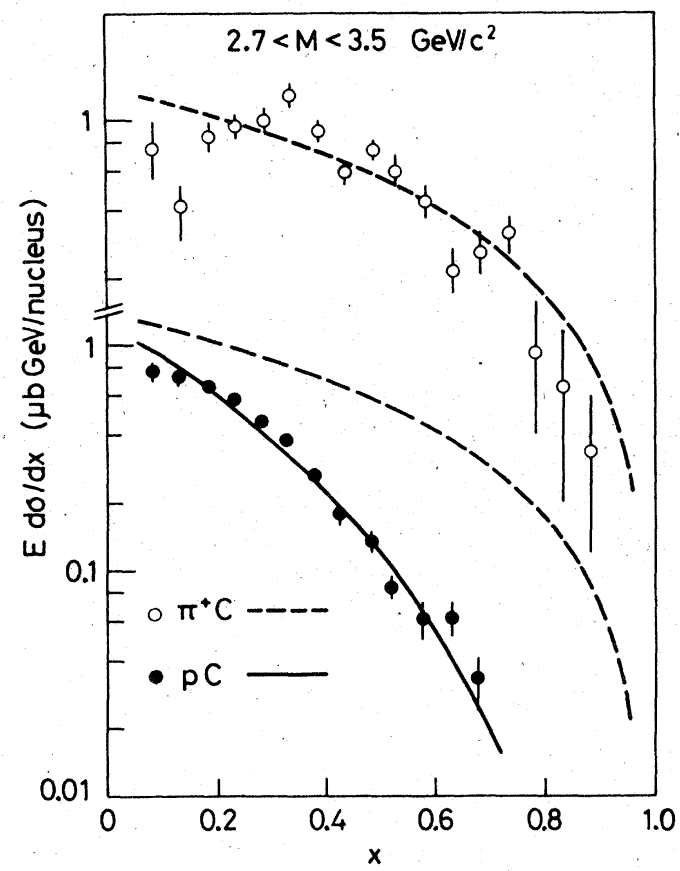

FIG. 17. Comparison of the relation of Eq. (32), $\left.\left(\phi / \pi^{*}\right)\right|_{\psi} \simeq 0.89 .(1-x)^{2.11}$, with the data of Ref. 31. Using an an interpolation of the $\pi^{*}$ data (dashed line) we predict the magnitude and energy dependence of the $p$ data (solid line). tent of the target. Analogously, experimental deviations from the predictions presented in this section could be a sign of the presence of charmed quarks in the initial state wave function. We neglect them at present energies as their presence would be signaled by a sharpening of the $x$ dependence at $M=M_{\psi}$. It would simply reflect the steeper $x$ dependence of the charmed quarks annihilating into a $\psi$. This is not observed. ${ }^{31}$

We close this section by making a detailed comparison of dilepton data obtained with meson and baryon beams (see Tables I and II and Figs. 14 and 15). Although the predictions that

$$
\begin{aligned}
& \left.\frac{p}{\pi^{+}}\right|_{\psi}=\left.\frac{p}{\pi^{-}}\right|_{\psi} \simeq 0.38, \\
& \left.\frac{p}{\pi^{+}}\right|_{\gamma} \simeq 0.82, \\
& \left.\frac{p}{\pi^{*}}\right|_{\gamma} \simeq 0.31
\end{aligned}
$$

are in agreement with the data, ${ }^{31}$ one can sharpen the predictive power of the model by taking into account the fact that one expects valence quarks in a proton to be suppressed by a factor $(1-x)^{2}$ relative to those in a meson beam. ${ }^{35}$ This correction yields

$$
\left.\frac{p}{\pi^{+}}\right|_{\psi} \simeq \frac{1.24(1-x)^{3.44}}{1.39(1-x)^{1.33}} \simeq 0.89(1-x)^{2.11}
$$

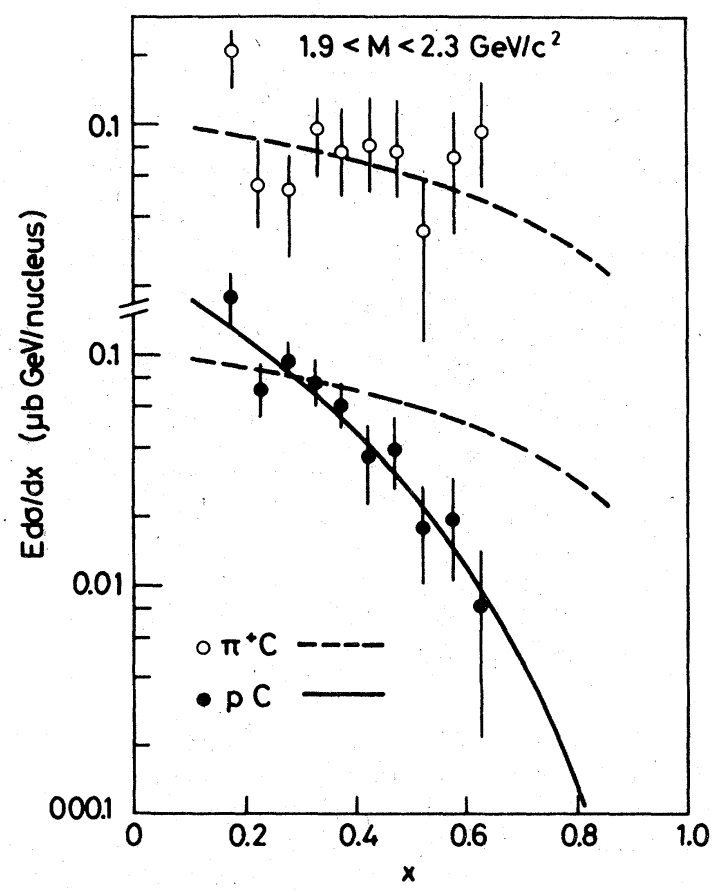

FIG. 18. Same as Fig. 17 for the relation of Eq. (33): $\left.\left(\phi / \pi^{+}\right)\right|_{\gamma} \simeq 2.29(1-x)^{2.45}$. Data are from Ref. 31 . 
and

$$
\left.\frac{p}{\pi^{+}}\right|_{\gamma} \simeq \frac{0.24(1-x)^{3.25}}{0.1(1-x)^{0.8}} \simeq 2.29(1-x)^{2.45},
$$

where we have actually taken the $x$ dependence for the different beam particle types directly from experiment. ${ }^{31}$ We notice that the expected $(1-x)^{2}$ suppression is approximately exhibited by the data and that integration over $x>0.15$ for the $p$ and $\pi^{+}$rates separately in Eqs. (32) and (33) recovers Eqs. (29) and (30), respectively. In Figs. 17 and 18 we show a comparison of Eqs. (32) and (33) with the data. ${ }^{31}$ Note in Fig. 18 the crossover in the $\left.\left(p / \pi^{+}\right)\right|_{\gamma}$ ratio at $x \simeq 0.35$, predicted by Eq. (33). Because of the reduced $\left.\left(p / \pi^{+}\right)\right|_{\psi}$ ratio, this crossover should disappear on resonance. It does, as shown in Fig. 17.

We would like to point out that a value $\epsilon=\frac{1}{4}$ for the relative abundance of sea compared to valence quarks, corresponds to a suppression of the role of the valence quarks by a factor $5 \sim 10$ compared to what one would have expected from a fullfledged Drell-Yan calculation.

\section{AN AFTERTHOUGHT}

The most important message of our generalized CVC proposal (Fig. 1) is that the experimentally observed flattening at high mass of the dilepton yield in hadron collisions signals a corresponding flattening of the hadroproduction cross section at high produced masses. The implication is that the approximately exponential mass dependence of the cross section for low values of $M$, that inspired the statistical model, corresponds to a local behavior. The $M$ dependence will flatten out for in- creasing $M$ values, with a trend similar to the $p_{T}$ dependence of $\pi$ inclusive cross sections. The message is that new quark flavors and weak bosons can be probed by future hadron machines. In fact CVC predicts cross sections in the $10^{-5}-\mathrm{mb}$ range for weak bosons provided $\sqrt{s} \gtrsim 300 \mathrm{GeV}$. The same result can be obtained in a two-line calculation using the $\Gamma / M^{3}$ scaling relation of Eq. (18). General arguments involving the conserved-vectorcurrent hypothesis lead to ${ }^{36}$

$$
\Gamma_{W}=\frac{G M_{W}^{3}}{3 \pi \sqrt{2}} R_{1}
$$

where $R_{1}$ is the isovector part of $\sigma\left(e^{+} e^{-} \rightarrow\right.$ hadrons $) /$ $\sigma\left(e^{+} e^{-} \rightarrow \mu^{+} \mu^{-}\right)$. For $M_{W} \simeq 60 \mathrm{GeV}$ we obtain from Eqs. (18) and (34) that

$$
\sigma_{\mathrm{W}}(\sqrt{s}) \simeq \sigma_{\psi}\left(\frac{M_{\psi}}{M_{W}} \sqrt{s}\right)
$$

where we have used $R_{1} / 2 \sqrt{2} \sim 1$. We therefore obtain again $\sigma_{W} \simeq 10^{-5} \mathrm{mb}$ for $\sqrt{s} / M_{W} \simeq 4$.

\section{ACKNOWLEDGMENTS}

Discussions with J. Ellis, M. K. Gaillard, and D. Nanopoulos are acknowledged. We thank J. Prentki and R. J. N. Phillips for the hospitality extended to us at CERN and the Rutherford Laboratory, respectively, where this work originated. This research was supported in part by the University of Wisconsin Research Committee with funds granted by the Wisconsin Alumni Research Foundation, and in part by the Energy Research and Development Administration under contract No. EY-76-C-02-0881, COO-881-3.
+ On leave of absence from Department of Physics, Kyoto University, Kyoto 606, Japan.

${ }^{1}$ H. Fritzsch, Phys. Lett. B67, 217 (1977).

${ }^{2}$ F. Halzen, Phys. Lett. B $\overline{69}, 105$ (1977).

${ }^{3}$ S. W. Herb et al., Phys. Rev. Lett. 39, 252 (1977).

${ }^{4}$ Y. Yamaguchi, Nuovo Cimento 43, $\overline{199}$ (1966); L. M. Lederman and B. G. Pope, Phys. Rev. Lett. 27, 765 (1971); L. M. Lederman and D. H. Saxon, Nucl. Phys. B63, 315 (1973).

5 For recent reviews, see D. Sivers, in New Fields in Hadronic Physics, proceedings of the XIth Rencontre de Moriond, Flaine-Haute-Savoie, 1976, edited by J. Trân Thanh Vân (CNRS, Paris, 1976); F. Halzen, in Proceedings of the International Conference on Production of Particles with New Quantum Numbers, Madison, Wisconsin, 1976, edited by D. B. Cline and J. J. Kolonko (Univ. of Wisconsin, Madison, 1976), p. 123; D. Hitlin, Summer Institute on Particle Physics, SLAC, 1976 (unpublished); K. C. Stanfield, in Particles and Fields '76, proceedings of the Annual Meeting of the Division of Particles and Fields of the American Physical Society, Brookhaven National Laboratory, edited by H. Gordon and R. F. Peierls, (BNL, Upton, N.Y., 1976), p. B1.

${ }^{6} \mathrm{~A}$. Donnachie and P. V. Landshoff, Nucl. Phys. B112, 233 (1976) and references to related work.

${ }^{7}$ We denote as $b, t$ the $q=-\frac{1}{3}, \frac{2}{3}$ components of a new quark doublet with an approximate mass of $5 \mathrm{GeV}$ associated with the $\Upsilon(9.5)^{3}$.

${ }^{8}$ T. K. Gaisser, F. Halzen, and E. A. Paschos, Phys. Rev. D 15, 2572 (1977).

${ }^{9}$ T. K. Gaisser et al. (Ref. 8) previously proposed this rule and in fact predicted the $\Upsilon(9.5)$ cross section at the presently observed level.

${ }^{10}$ See e.g., E. Eichten and K. Gottfried, Phys. Lett. B66, 286 (1977).

${ }^{11} \mathrm{~J}$. Ellis, M. K. Gaillard, D. V. Nanopoulos, and S. Rudaz, CERN Report No. CERN TH. 2346, 1977 (unpublished).

${ }^{12}$ T. Appelquist and H. D. Politzer, Phys. Rev. D 12 , 1404 (1975).

${ }^{13} \mathrm{~S}$. Brodsky, work presented at the Rencontre de 
Moriond, Flaine-Haute-Savoie, 1977 (unpublished).

${ }^{14}$ Brodsky (Ref. 13) has discussed the application of similar ideas to the production of light quarks.

${ }^{15}$ T. Appelquist and H. D. Politzer, Phys. Rev. Lett. 34, 43 (1975).

${ }^{16}$ A. De Rújula and S. L. Glashow, Phys. Rev. Lett. 34, 46 (1975).

${ }^{17}$ M. J. Corden et al., Phys. Lett. 68B, 96 (1977).

${ }^{18}$ Some diagrams of higher order in $\alpha_{g}$ might actually not be negligible for charm production.

${ }^{19}$ Detailed calculations are forthcoming: D. Sivers and B. Combridge (private communication). The two-gluon diagrams have been previously considered in the context of models producing $\psi$ 's via production and radiative decay of $\chi$ states, see e.g., M. K. Gaillard, B. W. Lee, and J. L. Rosner, Rev. Mod. Phys. 47, 277 (1975); F. Halzen, University of Wisconsin Report No. COO-501, 1976 (unpublished); Ref. 5; C. E. Carlson and R. Suaya, Phys. Rev. D 14, 3115 (1976); S. D. Ellis, M. B. Einhorn, and C. Quigg, Phys. Rev. Lett. 36, 1263 (1976); L. M. Jones and H. W. Wyld, Jr., Phys. Rev. D (to be published); M. Gluck, J. F. Owens, and E. Reya, Florida State University Report No. FSU HEP 770810, 1977 (unpublished). The cross section is given by

$$
\begin{aligned}
\frac{d \sigma}{d x}= & \frac{8 \pi^{2}}{M_{\mathrm{\chi}}{ }^{3}} \frac{\Gamma(\chi \rightarrow 2 g) \Gamma(\chi \rightarrow \psi \gamma)}{(\chi \rightarrow \text { all })} \frac{M_{\chi}{ }^{2}}{M_{\mathrm{\chi}}{ }^{2}-M_{\mathrm{\chi}}{ }^{2}} \\
& \times \int \frac{\tau d x}{x^{2}+\tau} G(x) G(\tau / x),
\end{aligned}
$$

where $\tau=M_{\mathrm{X}}{ }^{2} / \mathrm{s}$ and $G(x)$ is the longitudinal-momentum distribution of gluons inside a proton (see e.g., Ref. 21). This result has to be compared with Eq. (14) and is, in the context of QCD, expected to contribute to $\psi$ production at higher energies, as discussed in the text.

${ }^{20}$ Evaluated at $s=4 M_{C}{ }^{2}, t=u=-M_{C}{ }^{2}$.

${ }^{21}$ We used the structure functions of A. J. Buras and K. J. F. Gaemers, CERN Report No. TH. 2322, 1977 (unpublished).

${ }^{22}$ It is of course beyond the scope of this model to describe possible purely diffractive production sources of heavy particles. They are not expected to play an important role at high energies.

${ }^{23}$ T. K. Gaisser and F. Halzen, Phys. Rev. D 11, 3157 (1975).

${ }^{24}$ For a review of the data, see: H. Frisch, in Particles and Fields'76, proceedings of the Annual Meeting of the Division of Particles and Fields of the American Physical Society (BNL, Upton, N.Y., 1977), p. F59; L. Lederman, ibid., p. A31; P. Piroué, ibid., p. A1. ${ }^{25}$ Back-of-the-envelope calculation can be done using the following parametrizations;

$$
d \sigma^{\gamma} / d M^{\prime}=\left(8.84 \times 10^{-3}\right) e^{-3.5 M^{\prime}}+\frac{2 \times 10^{-4}}{M^{\prime 5}}
$$

or for $M^{\prime}>5$

$$
\left.\frac{d \sigma^{\gamma}}{d M^{\prime} d y}\right|_{y=0}=\left(1.89 \times 10^{-6}\right) e^{-0.98 M^{\prime}} .
$$

Units are $\mathrm{mb}$ and $\mathrm{GeV}$ throughout this paper. These parametrizations "subtract" resonance structure from the data. They agree well with the data interpolations of Kajantie (see Ref. 26).

${ }^{26} \mathrm{~K}$. Kajantie, invited talk at the Budapest Conference, 1977 (unpublished).

${ }^{27}$ T. K. Gaisser and F. Halzen, Phys. Rev. D 14, 3153 (1976).

${ }^{28}$ The calculation is in fact not sensitive to the chosen interval in dilepton mass $M^{\prime}$.

${ }^{29}$ For $\Upsilon$ we again assume typical (Ref. 11 ) values $B \simeq 0.05$ and a direct hadronic width $\Gamma \simeq 10 \mathrm{keV}$. The calculation involves some model dependence because of the unmeasured $x$ dependence of $\Upsilon$ production.

${ }^{30} \mathrm{~F}$. Halzen and S. Matsuda, Rutherford Report No. RL-77-072-A, 1977 (unpublished).

${ }^{31}$ J. G. Branson et al., Phys. Rev. Lett. 38, 1331 (1977); ibid 38, 1334 (1977).

${ }^{32}$ Note, however, that we never have to assume the detailed dynamical structure of the Drell-Yan diagram (Ref. 6) as a simple convolution of two form factors . We only flirt with that idea discussing the $x$ dependence of beam particle ratios for dilepton production.

${ }^{33}$ We neglect the charmed-quark component in the original collision.

${ }^{34} \mathrm{As}$ a guide we take bins in a mass plot adjacent to the point $M=M_{\psi}$. This is an approximate procedure, however, because of the presence of other resonances (e.g., $\left.\psi^{\prime}\right)$.

${ }^{35}$ See, e.g., S. Brodsky and J. Gunion, in Proceedings of the VIIth International Colloquium on Multiparticle Reactions, Tutzing, 1976 (unpublished).

${ }^{36}$ L. F. Li and E. A. Paschos, Phys. Rev. D 3, 1178 (1971). See also J. D. Bjorken, Phys. Rev. 148, 1467 (1966). 